\title{
Urgences
}

\section{De la fiction romanesque à la poésie : " les miroirs éclatés du réel "}

\section{Max Roy}

Numéro 28, mai 1990

Le roman comme poétique

URI : https://id.erudit.org/iderudit/025592ar

DOI : https://doi.org/10.7202/025592ar

Aller au sommaire du numéro

Éditeur(s)

Urgences

ISSN

0226-9554 (imprimé)

1927-3924 (numérique)

Découvrir la revue

Citer cet article

Roy, M. (1990). De la fiction romanesque à la poésie : " les miroirs éclatés du réel ". Urgences, (28), 94-107. https://doi.org/10.7202/025592ar d'utilisation que vous pouvez consulter en ligne.

https://apropos.erudit.org/fr/usagers/politique-dutilisation/ 


\section{De la fiction romanesque à la poésie: «les miroirs éclatés du réel»}

\section{Max Roy}

La reproduction d'une toile de Gustav Klimt produit son effet sur la page de couverture d'un roman québécois contemporain. Ce portrait d'Émilie Flöge, qui aurait pu servir de «bristol» au peintre autrichien, permettrait-il d'entrer dans l'espace irréel de Miljours, le premier roman de Julie Stanton ${ }^{1}$ ? Il allie la manière décorative et la luminosité d'un visage. D'entrée de jeu, une double impression, d'excès et de réserve, de déjà vu et d'insolite... Pourquoi justement ce tableau? Le texte de la quatrième de couverture répond sans doute à une même fonction:

Avec une grande puissance d'évocation, Julie Stanton trace par petites touches souples et concises le portrait d'Anna Akhmatova dont la poésie s'entremêle ici à l'écriture romanesque, fiction et réalité se chevauchant à travers la figure énigmatique de cette Elena imaginée par l'auteure.

Voilà le lecteur prévenu sinon rassuré. Un programme de lecture lui est proposé qui ne le fera s'étonner ni d'une polyphonie ni d'une énigme. Le roman mettra en scène une "célèbre poète russe ". Il en fera le "portrait *, dit-on. Devenue personnage romanesque, Anna Akhmatova inspirera la narratrice. Des extraits de sa poésie apparaîtront ici et là, insérés dans la parole d'une autre, mais clairement signalés au moyen de l'italique.

La question semble entendue, car la rencontre des genres est manifeste et avouée. Ce roman parle de la poésie, mais se pourrait-il qu'il en fasse un usage transitif? S'y réaliserait-il une traversée des frontières de genres? Il se trouve, à tout le moins, des expressions poétiques dans ce roman. Quant aux brefs passages empruntés à l'œuvre d'Akhmatova, ils contribuent à donner au texte un ton particulier qui mériterait une étude. Il me semble, toutefois, que le roman convie à

1 Julie Stanton, Miljours, coll. « Fictions", Montréal, L'hexagone, 1989. Julie Stanton est connue comme journaliste et poète. Elle a également publié un récit autobiographique qui a pour titre: Ma fille comme une amante (Montréal. Leméac, 1981). 
autre chose, que sa clôture est une condition apparente de sa forme, mais non de sa matière. Miljours nous amène ailleurs. Je prends le risque de résumer sa trame narrative.

Habitée par le célèbre poème "Requiem * d'Anna Akhmatova, qu'elle se prépare à donner en concert, une cantatrice subit un grave accident d'auto et sombre dans le coma. Entre la vie et la mort, un autre univers s'ouvre à Marguerite Miljours. Elle rencontre une femme fascinante, Elena Annaïeva, dont elle devient vite amoureuse. Cette femme prétend être la fille de la poétesse russe Anna Akhmatova. S'entrecroisent des épisodes de leur vie, dans la Russie révolutionnaire puis stalinienne, et le développement de la relation entre Elena et Marguerite. Celle-ci, enfin, est ramenée à la réalité, contre son gré, à la suite d'une intervention chirurgicale. Mais le souvenir d'Elena est impérissable, et Marguerite va désormais "dans l'existence sans but ni raison " 2 . Elle n'a d'autre désir que celui d'Elena: "Ainsi Psyché privé d'Éros, mon âme n'aura de cesse de crier vers Elena. ${ }^{3}$

Le roman se compose de quinze courts chapitres où sont relatés, en alternance, les faits de la "vie extérieure — - de l'accident à la guérison de Marguerite, en passant par son séjour dans une clinique médicale et par l'évocation de son passé - et ceux de la "vie intérieure" - depuis la rencontre de Marguerite et d'Elena jusqu'à leur relation intime, en passant par le souvenir d'Akhmatova. Quoique complexe, la structure du récit ne produit aucune difficulté de lecture. La narratrice prend le soin de situer chaque épisode de son aventure. Les intitulés de chapitres sont même tout à fait explicites : "L'accident", "Où je la vois pour la première fois ", "Ầ la clinique, le soir du 16 février ", "Où elle réapparaît, inqualifiable transparence *, "À la clinique, la nuit du 16 février", "Où s'explique ce qu'elle cherchait à me dire», etc. Suivant un modèle canonique, le récit enchâsse plusieurs histoires, débutant et se terminant une année après l'événement central où la cantatrice "s'est abandonnée à l'insolite * 4 . Cet événement est à la fois une perte et une prise de conscience, car en quittant le monde réel, Marguerite Miljours 
atteint une réalité plus éblouissante, où se trouvent l'amour et la poésie. Selon elle, la "vraie vie " est ailleurs ${ }^{5}$ ! Le projet du récit est nettement exprimé dès les premières pages:

Mes amis m'ont longtemps écoutée puis ont cessé de s'intéresser à ce qu'ils appellent « le fantasme fantasmant de Marguerite $\nsim$. Ils sourient l'air absent. Le phénomène ne provoque plus leur curiosité. D'autres voix, des événements, ces choses qu'ils ont à faire les sollicitent. La vie les sollicite. La mienne m'attend ailleurs, là où s'est déroulée cette histoire qui n'en est pas une et je veux tout raconter comme si tout recommençait dans la vérité de ma nuit, les frontières abolies. Je raconterai les paysages, les visages, leur empreinte sur le cœur et la quête du cœur, l'énigmatique présence de cette femme sous mes paupières, ce qu'il en reste. Une femme pour eux fictive mais ils se trompent, quelque part Elena marche près de moi. 6

Les "frontières abolies", ce sont tout aussi bien celles du monde extérieur (le physique, le public) et du monde intérieur (le psychique, l'intime) que celles du réalisme et du fantastique, car ce qui est vécu dans «l'onirisme" n'est pas moins ressenti comme vrai et concret. Un passage, en particulier, le démontre:

\section{Passent deux silhouettes}

Elles pourraient être irréelles, la simple illusion d'un esprit fiévreux ou encore l'imperceptible mouvement de l'air dessinant des ombres. Deux silhouettes pourtant s'installent vraiment au centre de mon cil. L'œil d'abord leur donne une dimension excessive, des paillettes d'or brouillent l'exact pourtour des corps car ce sont des corps, ils ont le mouvement de ce qui bouge charnel, des gestes de vivants. Puis une fois le regard apprivoisé, les dimensions rejoignent le réel, s'atténuent. Rien de plus vrai alors que ce couple. Une histoire va débuter, onirique, dans l'onirisme où je flotte tout entière, aveugle, sourde à ce qui n'est pas ce vertigineux paysage, cette limpidité d'une conscience à l'aftût. 7

5 Cette formule évoque inévitablement un tout autre roman qui faisait le proces de la poesie, d'une certaine image de la poesie, mais avec lequel Miljours n'a rien à voir.

6 Miljours, p. 12.

7 Ibid., p. 18. 
Cette insistance à nier l'illusion, la mystification, se répercute sur plusieurs plans. Ainsi, les références spatio-temporelles se multiplient parfois superposées. Tandis qu'elle repose dans une clinique de Nice, en 1981, Marguerite voit Elena pour la première fois à Agadir, vers 1930. En outre, le récit d'Elena la conduira en bien d'autres lieux et époques. Cela se traduit, sur un plan actoriel, par la coexistence de personnages romanesques et historiques. Les uns gravitent autour de la cantatrice Marguerite Miljours - un musicien, une amie d'enfance, un imprésario, un ex-amant, des frères, des médecins - ou du mannequin Elena Annaïeva - un photographe, des amis, un père présumé... Les autres, convoqués par le récit d'Elena, sont liés, plus ou moins directement, à la vie de la poétesse russe, Anna Akhmatova. Plusieurs d'entre eux sont également poètes ou artistes: Blok, Mandelstam, Goumiliov, Maïakovski, Modigliani, Glebova-Soudeïkina... Comme le signale l'intitulé du huitième chapitre: :Où elle me conduit à Akhmatova 8 , Elena permet de passer d'une histoire à l'Histoire. La position médiane de ce chapitre ne tient pas du hasard. Bien que l'on reste toujours dans un univers construit de langage, la transition de l'imaginaire à l'historique est manifeste. Le temps du rêve devient l'espace du souvenir. Des dates, des lieux, des événements sont vérifiables. La fiction romanesque inclut des tranches de l'Histoire. Il en résulte un effet de réel accentué. Mais, plus encore, un paradoxe vaut la peine d'être souligné: c'est de l'intérieur que s'atteint l'extérieur. Le * fantasme " ou, si l'on veut, le " rêve * de Marguerite, conduit à la réalité d'Akhmatova.

Miljours n'est pas un essai historique, loin de là. Il n'est pas non plus une réflexion sur la poésie. Les références à une poétesse et à son œuvre sont motivées par un propos qui se résumerait aux rapports entre la réalité et la fiction - si ce n'est entre le conscient et l'inconscient. Ces rapports paraissent fondés sur une allégorie. L'état comateux de Marguerite Miljours pourrait être une autre forme de l'exil qui a été imposé à la poétesse Akhmatova. Condamnées au silence, l'une par l'étrange loi du corps, l'autre par la plus étrange loi de l'État, la cantatrice et la poétesse sont réduites à une vie intérieure. De telles analogies ne sont sans doute pas accidentelles; elles participent à l'efficace du discours romanesque. 
98

Ce qui est en cause ne tient pas seulement à des similitudes entre des situations, des lieux, des époques et des êtres, mais plus encore à leur rapprochement, voire à leur superposition, par une traversée de la fiction.

Il n'est pas étonnant, alors, que ce roman «personnel» allie un mode de narration autobiographique et un discours biographique. Quelle que soit la part de vérité de ce discours, le procédé me paraît particulièrement révélateur. En effet, le « récit rapporté * par Elena - qui comprend des épisodes de la vie d'Anna - devient vite le propre récit de Marguerite Miljours. Par un jeu de focalisation clairement signalé, la narratrice assiste à des événements d'un autre âge:

Est-ce l'engourdissement, cette gangue de chaleur nous enveloppant et le froid autour, est-ce cette confidence ou le jeu encore de ma mémoire secrète? en moi s'anime la steppe. La steppe s'éveille où me convie l'irrésistible attraction des destins. J'y suis tout à coup transportée. Voici le campement en son entier, ses tentes sous lesquelles passent et repassent des silhovettes. Voici l'abri, la mère et l'enfant. Quelle vision que celle-ci! Akhmatova jeune femme, Elena a l'âge tendre de la vie. Merci, ó dieux, de permettre à mon âme vagabonde de voyager au-delà du temps, de la logique, de l'Histoire qu'on raconte. Ces histoires sous ma paupière offrent leurs parcours uniques où s'agrandit l'horizon des destins officiels. ${ }^{9}$

Il faut souligner l'effet littéraire de ce subterfuge, qui est un élargissement du champ de vision. C'est comme si Schéhérazade s'incluait dans les récits qu'elle attribue à des tiers. Cependant, ce qui est raconté ici n'appartient pas plus à l'Histoire qu'Elena elle-même. Ainsi, sommes-nous plus que jamais dans l'univers de la fiction romanesque. En fait, d'après les documents officiels, Anna Akhmatova n'a eu qu'un fils. Cela n'est ni ignoré ni démenti dans le roman. C'est même plutôt l'occasion d'un aveu ou d'une mise au point et, surtout, d'un nouveau questionnement, comme en témoigne ce passage:

... étrange, étrange, ce profil de Lev n'apparaît pas dans la densité blanche de mes ombres comme si, là où j'erre, il n'eût jamais été de fils pour la poète mais seule Elena. Quelle vérité portent donc le visage et le nom d'Elena Annaïeva

Ibid., p. 76-77. 
qu'on ne retrouve pas dans l'Histoire? ailleurs, elle est pourtant venue à moi investie de sa filiation avec Akhmatova et j'y adhère comme en rêve on adhère aux àmes interchangées, aux identités factices, aux parcours peu orthodoxes, aux imbroglios merveilleusement déroutants. Les miroirs éclatés du réêl ! 10

À nouveau se constate une insistance dans ce discours. La fidélité au réel est tout aussi niée que l'était, ailleurs, le caractère illusoire de la rencontre avec Elena.

Si le roman laisse libre cours à l'imagination, il évoque aussi des événements qui ont effectivement marqué la vie de la poétesse et la Russie du vingtième siècle. Ceux-ci se rattachent évidemment à la révolution et aux excès du nouveau régime. Il est fait allusion aux purges staliniennes dont fut victime Goumiliov, le premier mari d'Akhmatova -, aux camps de concentration - où périt notamment Mandelstam -, au désespoir qui conduisit plus d'un au suicide parmi lesquels Maïakovski - et à la censure - qui fit d'Akhmatova une exilée de l'intérieur. Le plus souvent, un commentaire remplit, à cette occasion, une double fonction de dévoilement et d'explication. Mais il arrive également que le personnage Miljours se retrouve, comme précédemment, témoin d'une scène sinon mêlé à un événement notoire. C'est le cas de la première lecture publique d'Anna Akhmatova:

Ce soir de l'an mil neuf cent treize serait un soir particulier. Me voici donc inscrite avec les habitués du Chien errant dans le cours de l'Histoire durant l'époque qui précède les derniers sursauts de la Russio impériale.

[...] Ce soir, avec Aleksandre Blok, Mandelstam, Goumiliov et Maïakovski, ce sont ses premiers poèmes publics qu'elle lira dans quelques instants, Blok l'ayant précédée ici de son nom reconnu. Elle avance svelte, sa jupe étroite, son châle azuré, son large camée à la taille, une élégance négligée. Qui estelle, se demande-t-on, qui est cette femme dont la voix se fait elle-même poème, se fait chant ? 11

Les frontières de temps, d'espace et de vies s'abolissent dans cette vision. Il y a là une transgression des codes. De narratrice autobiographe, Miljours devient à la fois biographe

11 Ibid., p. 103. 
d'Akhmatova et "témoin oculaire* d'une histoire qui lui est pourtant étrangère.

Le récit oscille donc entre des situations imaginées et des fragments du réel. Cela n'a sans doute rien d'extraordinaire, mais pose tout de même la question du statut des matériaux romanesques. La route de Nice ou un bar à Agadir peuvent tout au moins dessiner une toile de fond vraisemblable pour une histoire que nous savons être une histoire de roman. C'est là leur fonction minimale. Mais qu'en est-il des personnages historiques et des épisodes de leur vie - fussent-ils en désaccord avec l'histoire officielle - qui sillonnent le roman? Qu'en est-il aussi de ces extraits du Requiem ou du Poeme sans héros d'Akhmatova que la narratrice intègre à son propre discours? Ils ont pour effet, sinon pour fonction, de multiplier les perspectives de lecture. L'attitude de réception qu'ils entraînent, en particulier, les distinguerait peut-être des autres matériaux du roman. L'usage romanesque d'événements réputés appartenir au monde réel accroît non seulement la vraisemblance - ce qui est le principe de l'illusion réaliste - mais aussi l'engagement du lecteur. Comme Elena * conduit * Marguerite, le roman conduit le lecteur à Akhmatova. Bien qu'il résiste à la tentation de la biographie, il invite à en apprendre davantage sur cette poétesse.

C'est certainement l'un des mérites - et l'un des enjeux avoués - du roman de Julie Stanton de nous mettre en rapport avec Akhmatova. De l'avis des spécialistes, celle-ci figure parmi les plus dignes représentants de la poésie russe du XXe siècle $^{12}$. Elle y occupe une place aussi importante que singulière. Anna Akhmatova a vécu de 1889 à 1966. Ses premiers poèmes, parus en 1910, lui ont immédiatement valu le succès. Nina Berberova écrit à son propos: «On lui doit le plus beau monument poétique dédié aux victimes des purges staliniennes, Requiem $* 13$. Incidemment, ce dernier fut publié à Munich, en 1963, et à l'insu de son auteure ${ }^{14}$. On retient

12 Parmi les ouvrages consacrés à la vie et à l'couvre d'Akhmatova, on pourra consulter: Amanda Haight, Anna Akhmatova. A Poetic Pilgrimage, New York/ London, Oxford University Press, 1976; Jeanne Rude, Anna Akhmatova, coll. a Poètes d'aujourd'hui ", n 179, Paris, Seghers, 1968; Lydia Tchoukovskaiia, Entretien avec Anna Akhmatova, Paris, Albin Michel, 1980.

13 Nina Berberova, C'est moi qui souligne, Arles, Actes sud, 1989, p. 510.

14 Une traduction française du poème a paru dans la revue Esprit, Paris, juillet 1964 , p. 4-13. 
d'elle, en particulier, Le rosaire (1914), Anno Domini MCMXXI (1922) et le Poème sans heros, qui a subi plusieurs remaniements depuis 1940 jusqu'à sa parution en 1962. La carrière poétique d'Akhmatova a été marquée par de longues interruptions. Elle s'est faite prudente et soumise après que son exmari Goumiliov ait été fusillé et que son fils ait été incarcéré. En 1946, sa poésie a été jugée décadente ${ }^{15}$. Exclue de l'Union des écrivains, l'auteure a été contrainte au silence pendant plus de dix ans. Réhabilitée peu à peu après la mort de Staline, Anna Akhmatova a connu la gloire dans les années soixante. Il est à noter que Miljours relate la plupart de ces faits ${ }^{16}$.

Sur le plan littéraire, l'œuvre d'Akhmatova est associée, à ses débuts, à l'école acméiste que fonde officiellement Goumiliov par un manifeste intitulé « l'Héritage symboliste et l'Acméisme „17. Celui-ci écrivait alors:

Prenant la place du symbolisme, voici un nouveau mouvement qui, quel que soit le nom qu'on lui donne - que ce soit Acméisme (du mot acmé, le plus haut degré de quelque chose, la fleur, la culmination) ou Adamisme (une conception de la vie virile et claire) - est un mouvement qui en tout cas demande un plus juste équilibre entre le sujet et l'objet que n'en avait le symbolisme. ${ }^{18}$

Julie Stanton fait connaître ce détail par un mot d'Elena: * avec d'autres de la Corporation des poètes, il a fondé l'Acméisme, un mouvement littéraire en opposition au symbolisme ${ }^{19}$. Outre Goumiliov, on tient Akhmatova et Mandelstam pour les meilleurs représentants de l'acméisme ${ }^{20}$. Mais

15 Un rapport de Jdanov au Comité central du Parti communiste suffit à condamner l'ceuvre. Il dénonçait * la poésie d'une petite dame enragée, qui s'agite fiévreusement entre son boudoir et son prie-dieu n en y relevant a des motifs érotico-amoureux entremelés à des motifs de tristesse, de nostalgie, de mort, de mysticisme, de désespoir " (cf. Michel Aucouturier, a Akhmatova (Anna) ", Encyclopædia universalis, Paris, 1980, vol. I, p. 538).

16 Signalons seulement la mort de Goumiliov et l'emprisonnement de Lev (Miljours, p. 50).

17 Apollon, $n^{\circ} 1,1913$. J'emprunte cette référence à Amanda Haight, op. cit.

18 Cité et traduit par Serge Fauchereau, dans L'avant-garde russe. Futuristes et acméistes, Paris, Belfond, 1979, p. 28-29.

19 Miljours, p. 73.

20 Nina Berberova, pour sa part, décrit ainsi ce mouvement littéraire: * ACMÉISME (appelé aussi ADAMISME, CLARISME ou NÉO-CLASSICISME), mouvement poétique (1910 1920) refusant l'orientation mystique et l'esthétisme du symbolisme, prónant la clarté de l'expression, le contact vivant avec la réalité sensible, l'importance du "métier " en matière de création poétique ", op. cit., p. 509. 
102

qu'est-ce que cela a à voir ici? Les citations d'Akhmatova portent-elles seulement la marque de cette esthétique? De fait, les commentateurs de la poésie d'Akhmatova s'entendent pour y reconnaître un grand dépouillement, une simplicité dans l'émotion et un caractère intimiste. Les traductions de l'œuvre semblent reproduire ces caractéristiques et, à défaut de la version originale, il faut s'y fier ${ }^{21}$. S'y observe un style direct qui prend parfois les accents de la langue parlée. La recherche formelle n'y est guère perceptible, même si, entre les premiers et les derniers poèmes qu'il nous soit donné de connaître, l'expression se fait plus raffinée et.le propos, plus obscur. La rigueur de la phrase n'empêche pas, en effet, les corrélations nombreuses et les changements subits de vision et de voix. On ne sait plus qui parle, qui, d'un je ou d'un $t u$, se découvre. C'est que les personnages et les époques sont voulus interchangeables. On ne peut manquer de noter, dans le Poème sans héros, les transpositions de cet ordre. *Le plus passionnant sans doute est le jeu perpétuel du moi et du toi ", soutient un critique 22 . Du reste, les personnages qui traversent le poème sont masqués, comme l'affirme cet incipit:

Le soir du Nouvel An 1941. Au lieu des invités que l'auteur attendait, les ombres du passé surgissent. Elles sont déguisées comme pour un bal masqué. ${ }^{23}$

Des «ombres» surgies du passé se retrouvent, également, dans Miljours. Les * masques», le " poète» et le «spectre», ce sont tantôt les contemporains d'Akhmatova, tantôt les compagnons de Marguerite ou d'Elena, tantôt encore Marguerite, Akhmatova et Elena elles-mêmes.

La superposition des histoires contribue, par ailleurs, à créer une polymodalité temporelle qui rappelle le Poème sans héros. Michel Aucouturier observe, à ce propos, la * présence obsédante du passé dans le présent qui matérialise le temps et cette présence du présent dans le passé qui se nomme le destin. " Il conclut que ces deux thèmes, * celui du temps

21 Julie Stanton précise que « Toutes les citations en italiques [dans Miljours] sont tirées de Anna Akhmatova, poème sans héros et autres couvres, traduit du russe et présenté par Jeanne et Fernand Rude, Maspero, coll. Voix, Paris, 1982 \%, op. cit., p. [113]. Cette édition, pourtant récente, n'est plus disponible.

22 Jean Blot, « Anna Akhmatova ", Preuves, n 133, mars 1962, p. 8.

23 Je me réfère à une première version française du Pö̀me sans héros, parue dans Preuves, op. cit., p. 7-13. 
comme absence et néant, celui de l'histoire comme destin et expiation, dominent désormais toute l'œuvre d'Akhmatova. ${ }^{24}$ Dans le roman Miljours, le temps "réel * est malheureux, puisqu'Elena en est absente; le temps *onirique*, en revanche, est une promesse d'émotions et de ravissement:

Je réalise soudain avoir perdu l'entière notion du temps au profit exclusif des émotions, l'anachronisme de la situation me frappe dans toute la stupeur de son éblouissant mystère, mais pourquoi tenter de l'élucider quand j'en suis à ce point ravie? qu'importe que l'on ait brouillé les horloges, les calendriers et que les époques ne correspondent plus à l'heure connue, qu'importe hier ou demain ou le passé infini, et que cette femme née au début du siècle m'apparaisse dans la jeune trentaine au moment où je la croise. Pourvu que je la retrouve ! 25

Il faut noter que « l'anachronisme * imprègne tout autant la narration que la diégèse. D'une façon générale, le temps verbal est le présent dans le récit onirique. Même les épisodes de l'enfance d'Elena ou les souvenirs d'Akhmatova sont racontés au présent. Ils sont rendus immédiats, contrairement aux situations qui se rapportent à la convalescence de Marguerite. Le monologue intérieur de Marguerite la ramène inévitablement au présent et au rêve. Plus qu'une idée, c'est un procédé caractéristique de la poésie d'Akhmatova qui est ici réactivé.

On peut établir bien d'autres rapports entre la poésie d'Akhmatova et le roman Miljours. Ainsi, dans la partie centrale du Poeme sans heros, apparait un personnage dont la narratrice - car ce poème est aussi un récit - dit qu'elle est son double ${ }^{26}$. La dédicace du poème nous révèle qu'il s'agit d'Olga Glebova-Soudeikina, une actrice et danseuse de renom ( Une femme très proche d'Akhmatova ${ }^{27}$, souligne Elena). Le poème naît du souvenir d'un jeune poète, qui s'est suicidé par amour pour elle. Cette figure tragique a peut-être son corollaire dans le roman. Ce pourrait être l'ex-amant de Marguerite ou celui d'Elena qui, l'un comme l'autre, ont sombré dans le

24 Michel Aucouturier, loc. cit.

25 Miljours, p. 22.

26 Amanda Haight insiste sur ce point: Most of all, Sudeykina, this beautiful and frivolous Petersburg "doll", who receives her guests in bed in a room full of birds, is Akhmatova "double". " (op. cit., p. 150).

27 Miljours, p. 75. 
104

désespoir. En l'occurrence, la vie amoureuse de Marguerite Miljours est peuplée de jeunes amants comme semble l'avoir été celle de Glebova-Soudeikina. Je n'insisterai pas là-dessus.

Il existe bien une duplicité voulue dans la vision du monde que propose le roman. Marguerite est cantatrice, Elena est mannequin. Comme Glebova-Soudeïkina, ce sont des femmes de la scène, éblouissantes en apparence. Ellesmêmes sont doubles. Marguerite Miljours souffre d'une infirmité physique. Cette disgrâce, dans la vie courante, devient pourtant une marque estimable de sa personnalité publique. La diva sait même en tirer avantage:

Tant de regards déjà sur moi. Regard d'abord de pitié pour Marguerite l'infirme quand enfant puis adolescente désespérément révoltée je devais les affronter là où j'allais, alors qu'ils se détournaient embarrassés vers d'harmonieuses beautés. Mais pour Miljours parée des feux de la scène, Marguerite de gloire et de pouvoir, les regards sont devenus ceux de l'envie montant des salles obscures, ceux conquérants des hommes sur a la cantatrice au pied bot . évoluant de cette démarche pour eux touchante maintenant qu'ennoblie par des atours de reine ou de pure jeune fille, et l'œillade porteuse d'invites parfois chez une femme à cause de cette voix plus qu'une voix, plus qu'un chant, plus qu'une aria mais la souffrance transcendée rejoignant chez elle quelque capitale douleur. 28

Un regard, un sourire, ce mouvement de la taille, l'insidieuse séduction d'une boiteuse à qui il faut offrir le bras mais qui le choisit entre cent tendus vers elle. Je refermais sur les maris le double piège de la force et de la faiblesse conjuguées, de la grâce dans la disgrâce [...]. ${ }^{29}$

Pour sa part, Elena apparaît comme * une femme qui marche avec ce pas aérien de celle qui marche au loin " 30 . Mais cette légèreté, cette grâce, a aussi son envers. Elena est habitée par le chagrin. Son enfant est mort-né. C'est là son secret qu'elle est venue partager avec une * âme jumelle*.

Par-delà leur propre dualité, Elena et Marguerite se rejoignent dans *le mystère des multiples consciences qui se 
cherchent sans le savoir pour enfin se trouver sans l'avoir cherché ${ }^{31}$. Ce rapprochement n'est pas seulement le fait d'une complicité amoureuse, s'il faut en croire l'une et l'autre: "En marge et doubles nous voici de connivence, âmes jumelles ${ }^{32}$, pense Marguerite. «[...] Elena, dit-elle encore, cette zone énigmatique en toi voilà ma turbulence, ma marée montante, ce déferlement qui me cherchait depuis des siècles, tu es ma vie reconnue, tu es. " ${ }^{33}$ Ces mots pourraient être ceux d'Elena: "en moi, tu te tiens sans doute depuis l'enfance mais aujourd'hui seulement je te trouve ${ }^{34}$, déclare-t-elle. Et ailleurs :

La vie est une allée blanche où l'on marche double et je marche vers toi Marguerite mon écho, malgré mon désir d'enfant qui ne peut s'accomplir à travers toi. Mais puisqu'un enfant m'a un jour habitée dont l'esprit grandit chaque jour en moi, ce désir je le dépose à tes pieds comme gage de mon amour, le nôtre si tu le veux sera de nous enfanter l'une l'autre jusqu'à ce que l'une par l'autre chacune accède à sa propre fécondité. 35

La proximité d'Elena et de Marguerite repose moins sur un destin commun que sur une même sensibilité qui se traduit dans leur récit respectif comme dans leur dialogue. Les discours s'échangent mais ne sont pas confondus pour autant.

À titre de narratrice, Marguerite Miljours demeure étrangement lucide. "Deux femmes, et si c'était la même? " 36 , suggère-t-elle, comme pour s'y retrouver ou pour rappeler un thème de la poésie d'Akhmatova. S'agirait-il d'un dédoublement ou d'une projection de l'inconscient? L'identité, en tout cas, s'atteint peut-être sur l'axe du désir par la rencontre de pulsions contraires. Ainsi, jusqu'à cette aventure onirique, les amours de Marguerite ont été aussi diverses que passagères. Même s'il n'est qu'un rêve, son amour pour Elena est absolu. Mais c'est une pulsion de mort qui l'habite, lorsque, précisément, on tente de la ramener à la vie:

[...] désespérément je tâchai de faire le vide en mon esprit, de reléguer dans un petit coin du cerveau, la diva, l'infirme, la 
femme plus amoureuse de l'amour que de l'amant, pour laisser émerger celle que je rêvais d'être pour Elena, dégagée et engagée. Puisque Isis n'avait pas répondu à mon appel, j'évoquai l'âme d'Akhmatova, ses propres mots sur la mort, j'étais au bord de quelque chose qui n'a pas de nom bien précis... une somnolence qui vous invite, une fuite loin de vous-même, la mort à laquelle j'aspirais car pressentant avec effroi que la science cette fois triompherait des dieux si les dieux ne s'interposaient pas très bientôt pour que je me fixe à demeure dans l'univers d'Elena. A ceux qui m'entouraient, je criais moi aussi, mais je vous préviens, je vis pour la dernière fois [...]. 37

Il faudrait en conclure qu'au réel et au fictif, à l'histoire et à la poésie, correspond la dichotomie plus fondamentale de la vie et de la mort. C'est par le Requiem que Marguerite passe d'un monde à l'autre, et c'est dans l'inconscient qu'elle trouve son propre absolu. Par la traversée de la mort (le coma) et de la fiction (la vision), elle atteint une autre vie qui lui paraît bien réelle.

La question du rapport entre la réalité et la fiction resterait toute théorique si ne pouvait être admis le rôle déterminant du regard dans le roman. Jai signalé, précédemment, la dualité des personnages d'Elena et de Marguerite, qui était de l'ordre du * paraître et de l'«être *. Sur un autre plan, le regard reste un mode de perception privilégié aussi bien dans la "vie extérieure " que dans la "vie intérieure " 38 . C'est un monde sensible, sensuel aussi, qui est exploré par le *fantasme». À cela, rien d'étonnant peut-être. Il faut remarquer, néanmoins, l'importance des détails physiques et le réseau des références visuelles qui permettent des rapprochements entre les personnages. Voyageant dans le temps et dans l'espace, Marguerite peut observer * le corps d'Akhmatova en tout point semblable à celui aujourd'hui d'Elena [...] 39. Dans leur apparence même, Marguerite Miljours et Anna Akhmatova ont aussi des points en commun. C'est, notamment, pour couvrir le regard, une "frange " tout à fait caractéristique 40 .

37 Ibid., p. 97. Les passages en italiques sont empruntés à l'œuvre d'Anna Akhmatova.

38 Je note, au passage, que l'environnement musical de Marguerite Miljours le còde en importance à la * luminosité n d'Elena.

39 lbid., p. 77.

40 Ibid., p. 30 et 68. 
Plus largement, leur élégance physique aurait su inspirer des peintres. Ainsi, au portrait d'Akhmatova par Modigliani aurait pu correspondre celui de Miljours par Klimt: " cette allure lyrique "Klimt l'aurait sûrement peinte, quelque chose chez elle de son Émilie Flöge” [...]. „41 Ce détail n'est pas gratuit, car le tableau de Klimt pourrait refleter la vision éblouissante d'Elena: « sa lumineuse beauté dans la glace 42 . Comme lui, le roman reste une interprétation du réel. $\dot{A}$ travers l'illusion, pourtant, "mille fois la certitude du visage d'Akhmatova au fond d'un miroir " 43 .

On aura sûrement compris que Julie Stanton s'inspire à la fois de la poésie et des souvenirs d'Akhmatova, et qu'elle utilise très librement ces matériaux ${ }^{44}$. Ils ont bien plus qu'une incidence thématique. Les divisions du roman, par exemple, rappellent le cycle des quinze poèmes qui composent le Requiem. Le discours d'Akhmatova sert, dans une certaine mesure, de modèle pour la construction du roman. Il est à signaler que son œuvre poétique représente une sorte de «journal intime " ${ }^{45}$ où sont emmêlés les souvenirs lointains et récents, et où le réel et l'imaginaire ont le même statut. «L'élément essentiel de la poésie d'Akhmatova, écrit Jeanne Rude, est l'inspiration autobiographique. „ 46

Bien sûr, le texte de Julie Stanton ne contient pas que des souvenirs d'Akhmatova. Ce n'est que l'un de ses aspects et l'un de ses matériaux. Il me semble suggérer une triple figure de l'amour-poésie: la mère, la fille et l'amante. Le roman se prêterait tout aussi bien à la mythanalyse qu'à une étude stylistique. Sa poétique consiste, à tout le moins, en une juxtaposition de récits, de temps et de regards. Le rêve côtoie la réalité comme l'Histoire, le fantastique. Ce roman serait-il un conte, une fable, qui ferait d'un poète au destin tragique un modèle? Le paradoxe, s'il en est un, est du ressort même de la fiction.

\footnotetext{
41 Ibid., p. 31.

42 Ibid., p. 26.

43 lbid., p. 14.

44 Est signalée de manière allusive, notamment, l'impression que fit la tour Eiffel, * rouillée et bancale ", sur Anna Akhmatova, en 1911 (ibid., p. 88).

45 Michel Aucouturier rappelle l'avis de B. Elkhenbaum sur ce sujet (op. cit.). II est à noter que ce théoricien du formalisme a consacré un ouvrage à la poésie d'Akhmatova (Potrograd, 1923). Cf. Thérie de la littérature. Textes des formalistes russes..., coll. « Tel Quel „, Paris, Seuil, 1965, p. 62.

46 Jeanne Rude, op. cit., p. 68.
} 